\title{
A MEMS CONTROLLED CAVITY OPTOMECHANICAL SENSING SYSTEM
}

\author{
H. Miao ${ }^{1,2}$, K. Srinivasan ${ }^{l}$, and V.A. Aksyuk ${ }^{l}$ \\ ${ }^{1}$ Center for Nanoscale Science and Technology, National Institute of Standards and Technology, Gaithersburg, \\ Maryland, USA \\ ${ }^{2}$ Maryland Nanocenter, University of Maryland, College Park, Maryland, USA
}

\begin{abstract}
A novel high optical Q Si microdisk photonic cavity sensor is integrated to read out mechanical motion of an electrostatically actuated cantilever achieving $4.6 \mathrm{fm} / \mathrm{Hz}^{1 / 2}$ displacement sensitivity for sub- $\mathrm{W}$ input power. The readout gain is electrically-tunable by a factor of 200 . Using cold-damping feedback we reduce the thermal mechanical vibration by a factor of $>1000$, flattening the mechanical transfer function, and demonstrate broadband sensing with $100 \mathrm{kHz}$ bandwidth, $>2.5$ times the fundamental mechanical frequency. With $<20 \mu \mathrm{m}$ footprint and intrinsic readout bandwidth of $>500 \mathrm{MHz}$, cavity optomechanics enables a practical and flexible MEMS sensing platform with a broad application range.
\end{abstract}

\section{INTRODUCTION Motivation}

Sensors based on micro and, more recently, nano-mechanical systems continue to develop rapidly. They enable a wide variety of applications, from ubiquitous, mature technology of MEMS accelerometers, to gyroscopes [1], rapidly advancing in performance, from biomedical sensing [2] and atomic force microscopy (AFM) $[3,4]$ with mechanically simple cantilevers, to measurement of torque from single flux quanta in superconductors [5] and spin-polarized electron currents [6], with specially designed, complicated micro- and nano-devices. Generally, shrinking the sensor size allows increasing the mechanical responsivity by making it more compliant, while simultaneously increasing the bandwidth by lowering the inertia, thus reaching higher mechanical gain-bandwidth products. The sensor precision is fundamentally limited by the thermal Langevin force noise, which is related to the sensor dissipation via the fluctuation dissipation theorem. This noise can be minimized by making the appropriate materials, design and processing choices to achieve very low dissipation rates, with mechanical quality factors $(\mathrm{Q})$ in the $10^{5}$ to $10^{6}$ range [7]. In addition to lowering the sensor input noise floor, high Q increases the sensor responsivity (mechanical gain) in a narrow region of frequencies near resonance. While the gain is increased proportional to the $\mathrm{Q}$, the bandwidth, over which this high gain is available, drops as $1 / \mathrm{Q}$.

In a typical mechanical sensor the change in the mechanical position, in response to the driving forces and the environment, is detected by some linear readout means, such as electrostatic, piezoresistive, piezoelectric or optical. To operate the sensor at the fundamental limit of precision imposed by the thermal mechanical noise, it is necessary that the noise introduced by the readout be below the mechanically amplified Langevin force noise, over the whole sensor bandwidth. While this requirement is easier to achieve for near-resonant operation due to very high mechanical gain in this narrow band frequency region, it is a much more challenging task for broadband sensing, where the readout precision is often the dominant limitation of the sensor performance.

The main motivation of this work is to develop and demonstrate a technique that simultaneously advances the on-chip MEMS position readout precision and bandwidth by several orders of magnitude. Such technology should be fully integrated, stable, compatible with many types of mechanical sensors, have small footprint, low power dissipation and require minimal external infrastructure. It should also allow tailoring the readout gain and dynamic range to specific application requirements. We realize such a technique based on integrated interferometric optical readout or cavity optomechanics, achieving $4.6 \mathrm{fm} / \mathrm{Hz}^{1 / 2}$ displacement sensitivity for sub- W input optical power.

Taking advantage of this high sensitivity, we use feedback and electrostatic actuation to reduce the thermal mechanical vibration of the sensing element by a factor of 1000, tune the mechanical transfer function to remove the resonant peak at the fundamental frequency of $\approx 39 \mathrm{kHz}$ and extend the measurement bandwidth to $100 \mathrm{kHz}$ ( $>2.5$ times the fundamental frequency). The input-referred sensor readout noise remains below the thermal mechanical noise over this whole frequency range.

\section{Approach}

The fundamental benefits of optical readout are small footprint and high sensitivity, both related to the wavelength of light, low power dissipation, absence of intrinsic thermal noise due to photon shot noise limited operation, and very high intrinsic bandwidth, related to the optical frequency. Optical cavities afford even higher sensitivity at the expense of slight reduction in bandwidth by capturing the photons and making them interact with the mechanical degree of freedom over the cavity lifetime.

Silicon nanophotonics provides a path to integrating such cavities on chip, achieving high optical quality factors up to $10^{6}$ in a system compatible with MEMS and CMOS processes and materials. Such fiber pigtailed and connectorized Si devices operate at telecommunication wavelength, where highly stable, low noise, compact and economical diode laser sources, as well as low noise detectors, are readily available.

We have chosen a relatively simple $\mathrm{Si}$ microdisk cavity design, where the light circulates along the microdisk edge in a whispering gallery mode. The optical connection to the cavity is provided through evanescent coupling from a fixed on-chip $\mathrm{Si}$ waveguide, which is end-fire coupled to conventional optical fibers glued into v-groves. However, in contrast to the previous cavity optomechanical work $[8,9]$, we are not sensing the mechanical breathing mode of the microdisk, because such mode is very stiff, high frequency and is not easily coupled to for sensing external mechanical forces without degrading the optical performance.

In a novel design, illustrated in Figure 1d, we separate the optical and the mechanical parts of the system. The microdisk itself does not move. Instead we introduce a flat $\mathrm{SiN}_{\mathrm{x}}$ dielectric ring suspended a short distance above the microdisk top surface and movable vertically toward and away from it. The ring is mechanically attached to a soft cantilever actuator, comprising the mechanically active part of the sensor. Because the microdisk is thin, a significant fraction of the optical mode power density is located outside the microdisk, in the evanescent tails below and above it, where it is affected by the ring. As the ring moves closer to the disk, a larger fraction of the optical power travels inside the ring dielectric, increasing the effective index and shifting the frequency of the optical resonance lower. With the ring a few hundred nanometers above the disk, the strong coupling between the cavity optical frequency and the ring position forms the basis for our sensitive optical readout. 

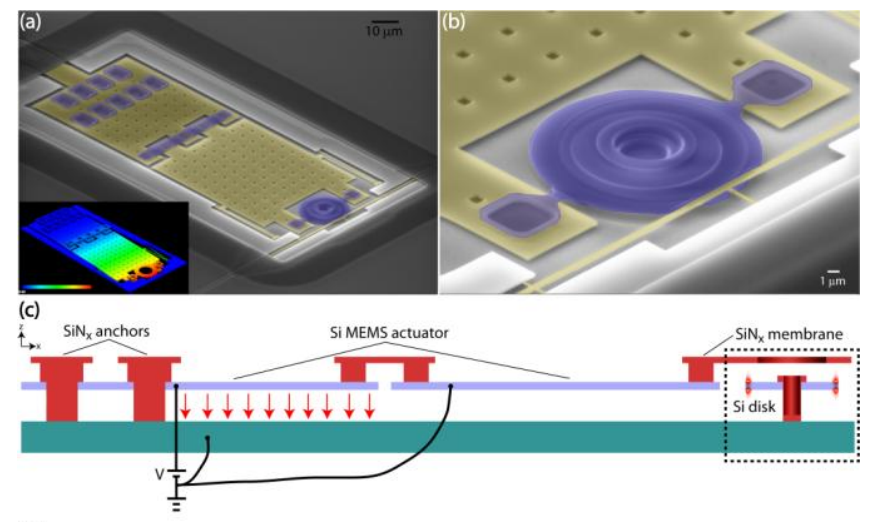

(d)

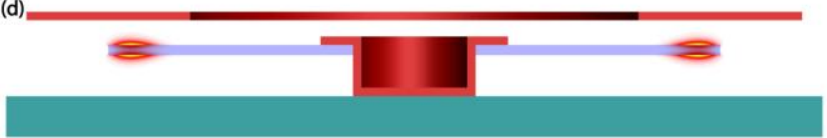

Figure 1: Device geometry. (a) SEM of the fabricated device. (inset) Finite element simulation of the fundamental mechanical mode of the mechanical structure. (b) Zoomed-in view of the sensor area. (c)-(d) $2 D$ cross-sectional illustrations of the device. The illustration shown in (c) is a cross section taken through the key elements of the mechanical sensor. In these cross-section illustrations, the $Z$ axis scale has been adjusted for clarity.

\section{EXPERIMENTAL}

\section{Design and fabrication}

Figure 1 shows the SEM and the illustrated cross sections of the sensing system. A 19 m outer diameter, $200 \mathrm{~nm}$ thick silicon nitride ring is placed within the evanescent field of a $15 \mathrm{~m}$ diameter, $240 \mathrm{~nm}$ thick silicon microdisk optical resonator, with a variable distance $\mathrm{Z}$ between them. The microdisk is fixed to the substrate via a silicon nitride anchor, while the ring is attached to a MEMS actuator. The actuator is a silicon cantilever consisting of two electrically separate parts that are mechanically joined by a dielectric silicon nitride bridge, with one side fixed to the substrate via silicon nitride anchors. Voltage is applied to the first part of the cantilever, while the substrate, $1 \mathrm{~m}$ below, is kept at the ground potential. The attractive electrostatic force bends the first part toward the substrate. The second part of the cantilever, maintained at the ground potential, serves as a mechanical lever arm to achieve a larger range of motion at the ring location. The resulting soft cantilever with the sensor ring has a fundamental mechanical bending mode (Figure 1a, inset) with a measured frequency of $\approx 38.5 \mathrm{kHz}$ and the stiffness at the sensor attachment point of $\approx 0.04$ $\mathrm{N} / \mathrm{m}$ from the FEM model.

The fabrication process was started with a silicon-on-insulator (SOI) wafer with $240 \mathrm{~nm}$ top silicon layer and $1 \mathrm{~m}$ buried oxide (BOX) layer. The device layer is patterned via electron beam lithography and dry etched down to the BOX layer to produce silicon microdisks, access waveguides and the actuators. The waveguide is linearly tapered down to a width of $125 \mathrm{~nm}$ at its end for low loss coupling with optical fibers. A sacrificial silicon dioxide layer $\quad(\approx 600 \mathrm{~nm}$ thick $)$ and a low stress silicon nitride layer $(\approx 200 \mathrm{~nm})$ are sequentially deposited in a low temperature oxide (LTO) and a silicon nitride low pressure chemical vapor deposition (LPCVD) furnace, patterned via optical lithography, and dry etched to form the nitride ring, nitride anchors and bridge and an electrical pad to ground the substrate. A photolithography, buffered oxide etch, and ion implantation (Boron) process was used to dope the first part of the cantilever and the pad to a doping level of $>10^{19} \mathrm{~cm}^{-3}$ for low contact resistivity. Another $1 \mathrm{~m}$ oxide layer was deposited. The wafer was annealed for $1 \mathrm{~h}$ at $1000{ }^{\circ} \mathrm{C}$ in an ambient $\mathrm{N}_{2}$ environment. Another photolithography, metal deposition, liftoff, dry etching and TMAH wet etching process was used to define the $\mathrm{V}$-grooves for fiber pigtailing. The device was finally placed in $49 \% \mathrm{HF}$ to undercut and release the movable structures, and critical point dried. Fibers are placed in the Vgrooves, aligned and cured into place with ultraviolet (UV) curable epoxy, with a fiber to fiber loss of $16 \mathrm{~dB}$.

\section{Transducer characteristics: static}

Figure $2 \mathrm{a}$ shows the electrostatic tuning of $Z$ and of the optical resonant wavelength as a function of the DC voltage applied to the actuator. The tuning of $Z$ is measured using a white light interferometric profiler. The resonant wavelength at various DC voltage values is measured via optical spectroscopy using a tunable wavelength laser. The inset of Fig. $2 b$ shows the transmission spectrum of one of the optical modes that is used for subsequent experiments. The linewidth of the optical mode is 4.1 $\mathrm{pm}$, corresponding to an optical Q of $3.8 \times 10^{5}$. To detect small motions of the cantilever we tune the laser to a set point on the linear slope of the optical resonance line (Figure $2 \mathrm{~b}$, inset). The change of the optical resonance frequency due to the mechanical motion causes a proportional change in the detected optical intensity. The sensor readout gain is proportional to the line slope given by the optical $\mathrm{Q}$ and the optomechanical coupling $g_{O M}=d \omega_{c} / d Z$, where $\omega_{c}$ is the optical resonance frequency. Figure $2 \mathrm{~b}$ shows the optomechanical coupling, calculated from Fig. 2a, which is increased by a factor of more than 200 (from $g_{O M} / 2 \pi=65$ $\mathrm{MHz} / \mathrm{nm}$ to $g_{O M} / 2 \pi=13.4 \mathrm{GHz} / \mathrm{nm}$ ) when the applied voltage is increased from $2.5 \mathrm{~V}$ to $8 \mathrm{~V}$, thus broadly adjusting the gain of the readout.
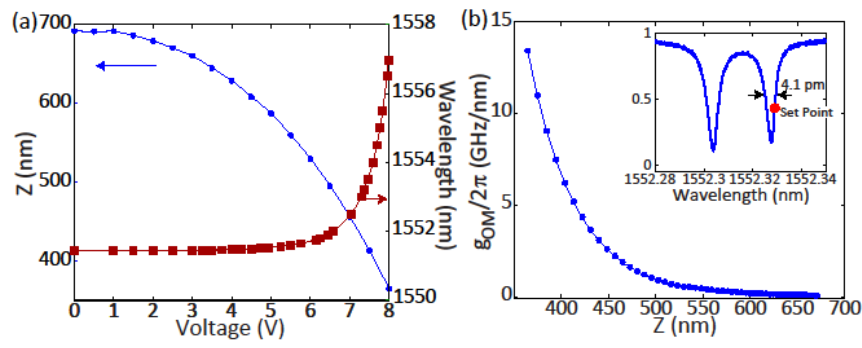

Figure 2: Static properties of the system. (a) Displacement and resonant wavelength as a function of bias voltage. (b) Optomechanical coupling as a function of displacement. Inset shows the transmission spectrum of the optical mode. In (a) and (b) the dots are the positions where the measurements were made.

\section{Transducer performance: dynamics and feedback}

We test the cavity optomechanical sensor by measuring the dynamics of the fundamental cantilever bending mode of the actuator. A CW light from a tunable wavelength laser is launched into the device, with an estimated optical power of $\approx 250 \mathrm{nW}$ in the waveguide, and $\approx 40 \mathrm{nW}$ out-coupled to a photodetector with a gain of $\approx 1.9 \times 10^{6} \mathrm{~V} / \mathrm{W}$ and a $3 \mathrm{~dB}$ bandwidth of $200 \mathrm{kHz}$. The wavelength is tuned to a pre-specified transmitted power set point as shown in the inset of Fig. 2b. A small motion of the cantilever results in a linear modulation of the transmitted optical power. The resulting photodetector voltage proportional to the mechanical displacement is recorded with an electrical signal analyzer.

We have measured two characteristics of our device (placed in a vacuum chamber with a pressure of $0.3 \mathrm{~Pa}$ ) under various 
conditions: the mechanical displacement noise spectral density and the sensor transfer function, shown in Fig. $3 a$ and $3 b$, respectively. The blue curves on Fig. $3 a$ and $3 b$ are taken under a fixed actuator DC bias voltage of $3 \mathrm{~V}$. To measure the transfer function, a small $\mathrm{AC}$ voltage with varying frequency is added to the DC bias, and the cantilever response is recorded in a lock-in measurement, normalized to the AC voltage. The black curve in Fig. 3a shows the noise background of the measurement.
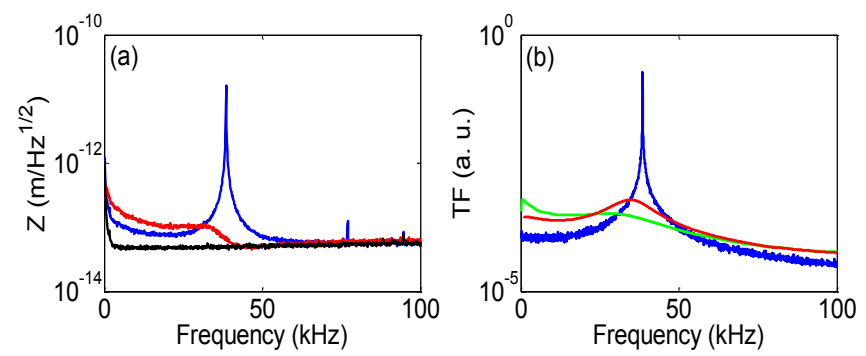

Figure 3: Device dynamics with and without cold damping. (a) Displacement noise spectra at 3 V DC bias with (red) and without (blue) feedback cooling. (b) Transfer functions (TF) corresponding to (a) (blue and red), and with higher feedback gain (green). Black curve in (a) shows the photodetector limited measurement sensitivity for both the undamped case and the damped case which are not distinguishable.

Although the high mechanical Q is desirable to reduce the thermal mechanical noise, the resulting highly non-uniform transfer function severely limits the sensor dynamic range if a broadband mechanical measurement is considered. We overcome this limitation by introducing a cold damping electrical feedback loop that modifies the sensor mechanical transfer function, reducing its variation across the frequency range. The red curves in Fig. $3 \mathrm{a}$ and $3 \mathrm{~b}$ show the noise spectrum and the corresponding transfer function at the same bias of $3 \mathrm{~V}$ with feedback cooling. The green curve in Fig. $3 \mathrm{~b}$ illustrates the transfer function with a higher damping gain setting, where the mechanical $Q$ is approaching 1. Considering the measured intrinsic $Q$ of $\approx 1400$, the root mean square thermal vibration amplitude of the mechanical mode is reduced by a factor of $>1000$. While the cold damping in itself does not reduce the input thermal force noise of the sensor, it does reduce any back-action that the sensor may exert on the physical system being measured by reducing the effective temperature of the sensor mechanical mode.

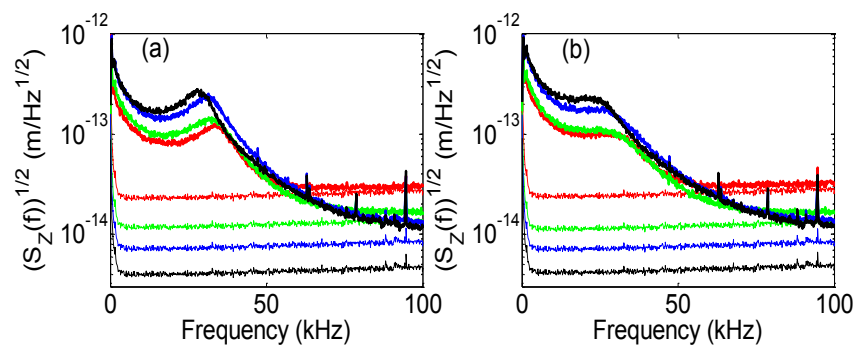

Figure 4: Displacement spectra at various bias voltages. (a) Under-damped case. (b) Critically damped case. The bias voltages for the red, green, blue and black curves are $4.5 \mathrm{~V}, 5.5 \mathrm{~V}, 6.25 \mathrm{~V}$ and $6.75 \mathrm{~V}$, respectively. The thicker lines show the measured ring displacement noise, the thinner lines are the corresponding photodetector limited background curves. The highest achieved readout sensitivity corresponds to the thin black lines.
The large reduction of the mechanical vibration of the sensor by the feedback allows working at increased DC bias, reaching higher readout gain and lower noise. Figure 4 shows the displacement noise spectra and the measurement noise backgrounds at increasing DC bias voltages. The degree of damping can be adjusted independently of the bias voltage, as illustrated by the two sets of curves in Fig. $4 \mathrm{a}$ and $4 \mathrm{~b}$. In addition to damping, our simple feedback choice also results in the reduction of the closed-loop mechanical stiffness. The displacement sensitivity increases by more than a factor of 15 when the bias voltage is increased from $4.5 \mathrm{~V}$ to $6.75 \mathrm{~V}$, and is limited by the photo detector noise to $4.6 \pm 0.6 \mathrm{fm} / \mathrm{Hz}^{1 / 2}$ at a bias voltage lever of $6.75 \mathrm{~V}$, which is only 2.3 times the standard quantum limit of the system.

\section{CONCLUSION \\ Summary}

We have realized a novel, fully-integrated and fiber-pigtailed high-precision optical readout of MEMS mechanical motion based on cavity optomechanics. The optical interferometric sensing of mechanical motion is achieved by evanescent coupling of a high $\mathrm{Q}=3.8 \times 10^{5}$ optical mode in a stationary Si microdisk optical cavity with a movable $\mathrm{SiN}_{\mathrm{x}}$ dielectric ring structure in close proximity above the microdisk. This design achieves large optomechanical coupling for high readout gain, while separating mechanical and optical parts of the system for independent optimization.

We demonstrate readout sensitivity below $5 \mathrm{fm} / \mathrm{Hz}^{1 / 2}$ limited by photodetector noise, with less than $1 \mathrm{~W}$ input optical power. The intrinsic bandwidth of our readout is above $500 \mathrm{MHz}$ limited by the photon lifetime in the optical cavity, while in this experiment it was limited by the detector bandwidth of $200 \mathrm{kHz}$. Our nanophotonic readout is highly stable and requires no optical alignment. The integrated actuator can tune the optomechanical readout gain by a factor of 200 .

While the total noise in our system is dominated by the thermal mechanical noise, it is noteworthy that the readout sensitivity approaches the standard quantum limit near resonance. From the practical perspective, the high sensitivity allows broadband mechanical sensing at frequencies much larger than the fundamental mechanical resonance. We have used the readout and the actuator in a feedback loop to flatten the mechanical transfer function to enable broadband measurements, dampen thermal mechanical motion of our sensor by a factor of $>1000$ and measure its vibration noise power spectrum over a broad mechanical bandwidth of $0 \mathrm{kHz}$ to $100 \mathrm{kHz}$, more than 2.5 times the fundamental mechanical resonance frequency of $\approx 39 \mathrm{kHz}$.

\section{Outlook}

We believe our integrated, stable MEMS sensing platform can enable a variety of scientific and technical sensing applications due to a combination of very high sensitivity, very high bandwidth and decoupling of mechanical and optical design parameters. The platform uses conventional MEMS materials and processes. The readout approach is suitable for high and low mechanical frequency systems with a broad range of stiffness and mass. The optomechanical gain can be fixed or widely tunable. For fixed optical gain operation the actuator may be unnecessary, enabling all-optical sensing. The ability to electrically tune the operating wavelength by up to several nanometers makes possible operation with practical and economical telecom fixed wavelength laser diodes. The small footprint, fiber readout and miniscule power consumption may enable novel sensor array applications. 


\section{REFERENCES}

[1] S. A. Zotov, M. C. Rivers, A. A. Trusov and A. M. Shkel, "Folded MEMS pyramid inertial measurement unit", IEEE Sensors Journal, 11, 2780-2789 (2011).

[2] O. Sahin, S. Magonov, C. Su, C. F. Quate and O. Solgaard, "An atomic force microscope tip designed to measure timevarying nanomechanical forces", Nature Nanotechnoloty. 2, 507-514 (2007).

[3] J.L. Arlett, E.B. Myers and M. L. Roukes, "Comparative advantages of mechanical biosensors", Nature Nanotechnology, 6, 203-215 (2011).

[4] K. Srinivasan, H. Miao, M. T. Rakher, M. Davanco and V. Aksyuk, "Optomechanical transduction of an integrated silicon cantilever probe using a microdisk resonator", Nano Letter, 11, 791-797 (2011).

[5] C. A. Bolle, V. Aksyuk, F. Pardo, P. L. Gammel, E. Zeldov, E. Bucher, R. Boie, D. J. Bishop and D. R. Nelson, "Observation of mesoscopic vortex physics using micromechanical oscillators", Nature 399 43-46 (1999).

[6] G. Zolfagharkhani, A. Gaidarzhy, P. Degiovanni, S. Kettemann, P. Fulde and P. Mohanty "Nanomechanical detection of itinerant electron spin flip", Nature Nanotechnology, 3 720-723 (2008).

[7] J. Yang, T. Ono and M. Esashi, "Surface effects and high quality factors in ultrathin single-crystal silicon cantilevers", Applied Physics Letters, 77, 3860 (2000).

[8] T. J. Kippenberg and K. J. Vahala, "Cavity opto-mechanics", Optics Express 15, 17172-17205 (2007).

[9] M. Eichenfield, J. Chan, R. M. Camacho, K. J. Vahala and O. Painter, "Optomechanical crystals", Nature, 462, 462, 78-82 (2009).

\section{CONTACT}

*V. A. Aksyuk, tel: +1-301-975-2867; vladimir.aksyuk@nist.gov 